\title{
Contribución al conocimiento de la orquideoflora de la Comunidad Foral de Navarra (Noreste de la Península Ibérica)
}

\author{
Estrella Robles Domínguez y Manuel Becerra Parra \\ C/ Pérez Goyena, 18-3ํㅡ. 31620 Huarte (Navarra).
}

\section{Correspondencia}

Manuel Becerra-Parra

e-mail: manuel.becerra.parra79@gmail.com

Recibido: 29 octubre 2020

Aceptado: 23 marzo 2021

Publicado on-line: 23 abril 2021

Editado por: Marta Recio Criado

\section{Resumen}

Se aportan algunas novedades corológicas y taxonómicas de 16 taxones de orquídeas de la Comunidad Foral de Navarra. Se proponen tres nuevos taxones, uno de ellos a nivel subespecífico y los otros dos hibridógenos.

Palabras clave. Orchidaceae, Dactylorhiza, Gymnadenia, Ophrys, Spiranthes, Platanthera, híbridos, corología, taxonomía.

\begin{abstract}
Contribution to the knowledge of the orchid flora from Navarra Foral Community (Northeast of the Iberian Peninsula)

New chorological and taxonomic items are provided for 16 taxa to the orchid flora from Navarra Foral Community. Three new taxa are proposed, one in subspecific level and the remaining two from hybrid origin.
\end{abstract}

Keywords. Orchidaceae, Dactylorhiza, Gymnadenia, Ophrys, Spiranthes, Platanthera, hybrids, chorology, taxonomy.

\section{Introducción}

La Comunidad Foral de Navarra está situada en el extremo norte del cuadrante noreste de la Península Ibérica, entre el valle del Ebro, al sur, y los conjuntos montañosos de los Pirineos y los Montes Vascos, al norte.

En este espacio, cuya superficie es de $10.391 \mathrm{~km}^{2}$, están representadas las dos grandes regiones biogeográficas peninsulares, la Eurosiberiana y la Mediterránea. Esto, junto a sus variadas condiciones climáticas, que a su vez están fuertemente influenciadas por su orografía, hacen de Navarra una de las regiones peninsulares con mayor diversidad de orquídeas. Así, están presentes 81 de los 116 taxones catalogados hasta la fecha en el ámbito ibero-balear (Benito Ayuso, 2017).

Desde 2017 venimos desarrollado un proyecto de investigación con objeto de profundizar en el conocimiento de la orquideoflora navarra. Como resultado de los distintos muestreos realizados hemos localizado varios taxones cuya presencia en la Comunidad Foral de Navarra se desconocía. Además, proponemos dos nuevos híbridos del género Ophrys, que según la bibliografía consultada no están descritos (Benito Ayuso, 2017), y separamos las poblaciones de Ophrys picta Link del norte peninsular como una subespecie distinta a la vista de una serie de caracteres morfológicos.

\section{Material y métodos}

De cada taxón se incluyen los siguientes datos: división administrativa (municipio y concejo en el caso de los municipios compuestos) y toponimia del paraje donde se ha recolectado (fuente de referencia: Geoportal de Navarra [https://sitna.navarra.es/geoportal]); coordenadas GPS (han sido tomadas con un Garmin GPSmap 62 en el datum ETRS89); ecología, teniendo como referencia para las asociaciones vegetales la clasificación fitosociológica del manual de hábitats de Navarra (Peralta et al. 2018) y geología (la litología ha sido consultada en el portal de la Infraestructura de Datos Espaciales de Navarra, [idena.navarra.es]; además, de la fecha de recolección u observación.

Hemos depositado todo el material recolectado en el herbario PAMP de la Universidad de Navarra. En el caso de Platanthera algeriensis Bat. \& Trab. y Spiranthes aestivalis (Poir.) Rich., por el reducido número de ejemplares que integran sus poblaciones y estar protegidos legalmente, no se ha recolectado material de herbario, pero en su defecto se incluyen fotografías realizadas en su medio natural. Éstas han sido realizadas con una cámara Nikon Coolpix B500.

Para la identificación de los ejemplares recolectados nos hemos basado en la sistemática empleada en las obras de Benito Ayuso (2017) y Aizpuru et al. (2000). 


\section{Resultados y discusión}

$\times$ Dactylodenia st-quintinii (Godfery) J. Duvign., Nouv. FI. Belg. Luxemb. France etc., ed. 3: 922 (1983).

[Gymnadenia conopsea (L.) R. Br. × Dactylorhiza fuchsii (Druce) Soó]

Material estudiado:

ESPAÑA. Navarra: Isaba, proximidades de la venta de Juan Pito. 30TXN7456. Herbazales sobre suelos encharcados; margas y limonitas; $1.150 \mathrm{~m}$. 30-VI2019. E. Robles \& M. Becerra (PAMP 56187) (Figura 1.1).

Observaciones: Las plantas observadas presentaban el labelo similar al de Gymnadenia conopsea, si bien el lóbulo central, que sobresale sobre los laterales, y las pequeñas máculas lineariformes de tonalidad rosa-violácea son caracteres propios de D. fuchsii. El espolón también es intermedio entre ambos taxones.

Novedad para el catálogo florístico de Navarra (Lorda, 2013). Tampoco nos constan citas bibliográficas para la Península Ibérica.

Dactylorhiza markusii (Tineo) H. Baumann \& Künkele in Mitt. Arbeitskreis Heimische Orchid. Baden-Württemberg 13:461 (1981).

[D. sulphurea (Link) Franco in Bot. J. Linn. Soc. 76:366 (1978)]

Material estudiado:

ESPAÑA. Navarra: Muruzábal, Frankomearra. 30TXN0330. $550 \mathrm{~m}$. Carrascal acidófilo con coscojas. 28-IV-2019. E. Robles \& M. Becerra (PAMP 56166).

ESPAÑA. Navarra: Uterga, Oianbazterra. 30TXN0329. $540 \mathrm{~m}$. Carrascal acidófilo con coscojas. 28-IV-2019. E. Robles \& M. Becerra (PAMP 56191). Observaciones: taxón que se distribuye por el NW de África, Cerdeña, Córcega, Península Itálica (Calabria) y Península lbérica (Sánchez Pedraja, 2005). Es muy rara en el cuadrante nororiental de la Península Ibérica, donde tan solo se conoce de La Rioja, Álava y Navarra. En esta última, a las poblaciones ya conocidas de Ayegui (Benito Ayuso, 2017) y Acedo (Amardeilh, 2012) hay que añadir éstas situadas en el pie de monte de la sierra del Perdón, en su vertiente sur. Aquí aparece en el sotobosque de encinares pertenecientes a la variante silicícola xerófila de los carrascales castellano-cantábricos de Spiraeo obovatae-Quercetum rotundifolia Rivas Goday ex Loidi \& F. Prieto.

Por su escasez ha sido recientemente incluida en el Listado Navarro de Especies Silvestres en Régimen de Protección Especial (Decreto Foral 254/2019, de 16 de octubre).

Una especie próxima es Dactylorhiza insularis (Sommier) Ó. Sánchez \& Herrero, que se distingue por las máculas de tonalidad rojiza, más o menos marcadas, que presenta en la base del labelo y su espolón recto -en $D$. markusii está curvado en su extremo-(Benito Ayuso, 2017).

Dactylorhiza × sooi (Ruppert ex Soó) Soó, Nom. Nov. Gen. Dactylorhiza: 10 (1962).
[Dactylorhiza alpestris $\times$ Dactylorhiza fuchsii (Druce) Soó]

Material estudiado:

ESPAÑA. Navarra: Isaba, barranco de Arrakogoiti. 30TXN7457. Prados subalpinos sobre suelos encharcados en los márgenes de una regata; margas y limonitas; 1.475 m. 6-VII-2019. E. Robles \& $M$. Becerra (PAMP 56184) (Figura 1.2).

Observaciones: El labelo es de una tonalidad intermedia y presenta el lóbulo central sobresaliente y las máculas lineariformes propios de Dactylorhiza fuchsii. Por su parte, el espolón es más próximo al que presenta este último taxón.

Novedad para el catálogo florístico de Navarra (Lorda, 2013). Tampoco nos constan citas bibliográficas para la Península lbérica.
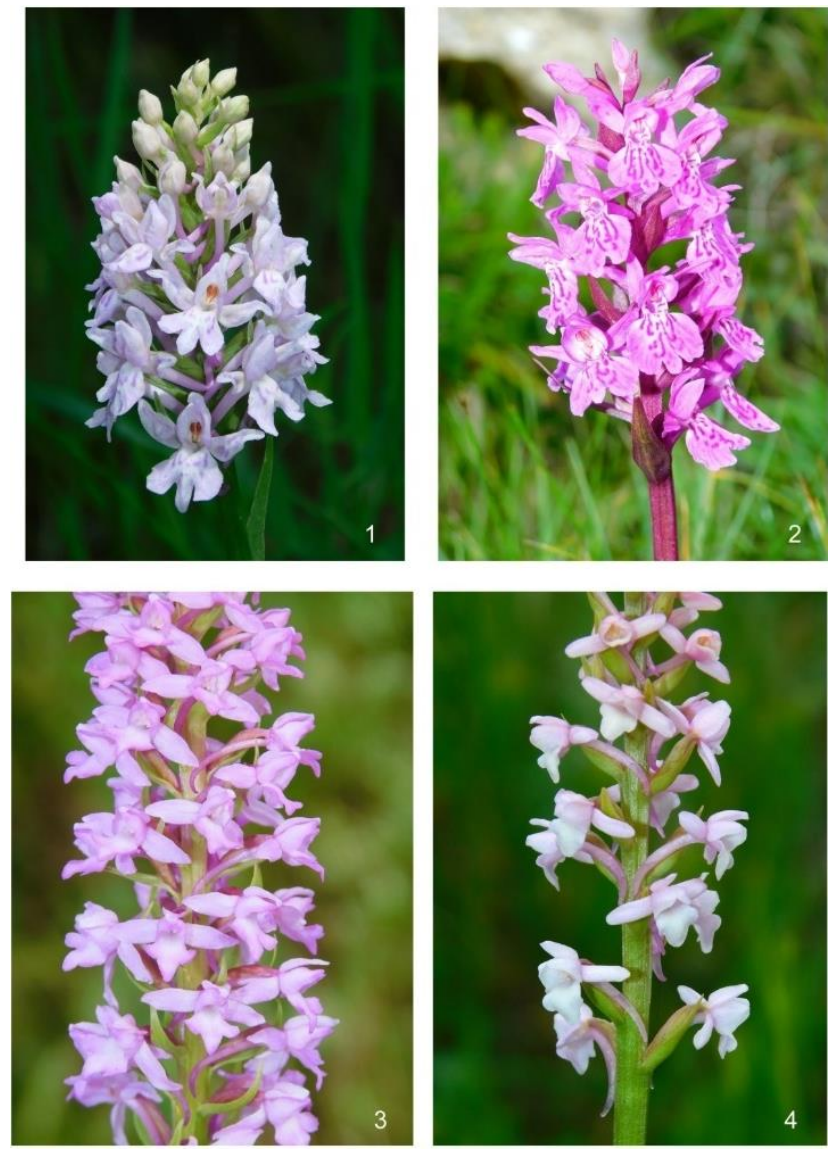

Figura 1. 1) × Dactylodenia st-quintini (Isaba, 30-VI-2019) 2) Dactylorhiza $\times$ sooi (Isaba, 06-VII-2019). 3) Gymnadenia densiflora (Ergoiena, 10-VI-2020). 4) Gymnadenia pyrenaica (Olazagutía, 10-VI-2020).

Figure 1. 1) $\times$ Dactylodenia st-quintini (Isaba, 2019/06/30). 2) Dactylorhiza $\times$ sooi (Isaba, 2019/07/06). 3) Gymnadenia densiflora (Ergoiena, 2020/06/10). 4) Gymnadenia pyrenaica (Olazagutía, 2020/06/10).

Gymnadenia densiflora (Wahlenberg) Dietrich in Allg. Gartenzeitung 7:170 (1839).

[G. conopsea subsp. densiflora (Wahlenb.) E. G. Camus, Bergon \& A. Camus in Monogr. Orchid. 327 (1908)]

[G. conopsea subsp. densiflora (Wahlenb.) E. G. Camus, Bergon \& A. Camus] 
Material estudiado:

ESPAÑA. Navarra: Isaba, proximidades de la ermita de Arrako. 30TXN7455. $940 \mathrm{~m}$. Herbazales en los márgenes de una regata, margas. 20-VII-2019. E. Robles \& M. Becerra (PAMP 56188).

ESPAÑA. Navarra: Ergoiena, concejo de Unanu, Batzarremendi. 30TWN7947. $605 \mathrm{~m}$. Prados de siega con Cynosurus cristatus, margas. 10-VI-2020. E. Robles \& M. Becerra (PAMP 57014) (Figura 1.3).

Observaciones: especie cuya entidad taxonómica no es reconocida por muchos autores, como es el caso de Flora iberica, donde se considera una forma de $G$. conopsea con la inflorescencia algo más densa y espolón relativamente corto (Aedo, 2005). Otros botánicos, sin embargo, la reconocen como un taxón independiente (Hermosilla, 2000; Benito Ayuso, 2017). Entre los caracteres morfológicos que lo separan de $G$. conopsea se encuentran su inflorescencia por lo general más densa; el lóbulo central del labelo que sobresale sobre los laterales y es agudo -en G. conopsea es obtuso-; su garganta frecuentemente surcada y de tonalidad más pálida, incluso blanquecina, que contrasta con el resto de la flor; y su espolón netamente más corto $(<13 \mathrm{~mm})$ (Benito Ayuso, 2017). A todo ello debemos añadir que en las dos localidades donde la hemos observado, donde comparte hábitat con G. conopsea, presenta una fenología más tardía, floreciendo en torno a dos o tres semanas más tarde que esta última especie.

Taxón euroasiático que en la Península Ibérica se circunscribe a las montañas del cuadrante noreste peninsular: Montes Vascos (Álava), Sistema Ibérico (La Rioja, Soria, Guadalajara, Teruel) y Pirineos (Gerona, Huesca y Navarra) (Benito Ayuso, 2017).

En la Comunidad Foral de Navarra es un taxón que parece ha pasado desapercibido. Lorda (2013) no la incluye en su catálogo florístico de Navarra mientras que Van der Sluys \& González (1982) incluyen un dibujo e indican su presencia en el valle de Belagoa. Con nuestras observaciones confirmamos su presencia.

Gymnadenia pyrenaica (Philippe) Giraudias, J. Bot. (Morot) 6:479 (1892).

[G. conopsea subsp. pyrenaica (Philippe) K. Richt, PI. Eur. 1:279 (1890)]; [G. odoratissima subsp. longicalcarata C.E. Hermosilla \& Sabando in Estud. Mus. Ci. Nat. Álava 10-11:123, 128 (1996)].

[G. conopsea subsp. pyrenaica (Philippe) K. Richt]; [G. odoratissima subsp. longicalcarata Hermosilla \& Sabando]

Material estudiado:

ESPAÑA. Navarra: Olazagutía, Mendiaundi. 30TWN6747. 595 m. Prados de siega con Cynosurus cristatus, margas. 10-VI-2020. E. Robles \& $M$. Becerra (PAMP 57015) (Figura 1.4.)

Observaciones: se distingue de los otros dos taxones del género Gymnadenia presentes en el ámbito peninsular por su menor porte, inflorescencia más corta y menos densa, con las flores poco coloreadas, presentando el labelo tonalidades rosáceas pálidas, en algunas plantas incluso de color blanquecino- rosáceo; el labelo está levemente trilobulado sobrepasando el lóbulo central a los laterales (Hermosilla \& Sabando, 1996). No obstante, el principal carácter morfológico que lo caracteriza es su espolón corto, de menos de $7 \mathrm{~mm}$, y que supera ligeramente la longitud del ovario. En cuanto a su fenología, florece unos 15-20 días más tarde que $G$. conopsea.

En lo referente al tratamiento taxonómico dado por los distintos autores a esta especie, Aedo (2005), en Flora iberica, considera que las plantas peninsulares se corresponden con G. odoratissima (L.) Rich. Con anterioridad, Hermosilla \& Sabando (1996) separan las poblaciones ibéricas como una subespecie distinta a la que denominan $G$. odoratissima subsp. longicalcarata C. E. Hermos. \& Sabando sobre la base de algunas diferencias en la longitud del espolón, tras estudiar material recolectado en las provincias de Burgos y Álava. No se detectó, sin embargo, que estos individuos de flores con espolón que apenas supera la longitud del ovario ya fueron descritos en las vertientes francesa de los Pirineos por el botánico francés Philippe en 1860 como Orchis pyrenaica Philippe y posteriormente recombinados en el género Gymnadenia por Giraudias en 1870 (Benito Ayuso, 2017).

La distribución de este endemismo hispanofrancés en la Península lbérica se circunscribe a la Cordillera Cantábrica (Asturias, Cantabria, León), Montes Vascos (Álava, Navarra), Pirineos catalanes y de manera puntual en el Sistema Ibérico (Soria) (Benito Ayuso, 2017).

En cuanto a la Comunidad Foral de Navarra, no nos constaba su presencia, si bien era de esperar al no ser rara en localidades alavesas muy próximas a la muga navarra, como es el caso del entorno de la localidad de Korres (Parque Natural de Izki). La única población localizada se sitúa en el pie de monte de la sierra de Urbasa, en su vertiente norte y no muy lejos del límite con Álava.

La referencia que Van der Sluys \& González (1982) hacen de $G$. odoratissima, en la que incluyen un dibujo donde sus caracteres morfológicos no se terminan de ver con claridad, hay que tomarla con reservas. En ninguna de las localidades enumeradas por ellos (partes altas de Belagoa, Minchate y Belabarce, montes de Iturgoyen) la hemos podido localizar.

El escaso número de individuos observado $(<100$ ejemplares) así como las posibles afecciones que pueda sufrir en el entorno donde crece (modificación del trazado de la carretera NA-7183 y posibles desbroces en épocas inadecuadas) hacen necesario algún tipo de actuación destinada a su conservación así como la propuesta al Gobierno de Navarra para su futura inclusión en el Listado Navarro de Especies Silvestres en Régimen de Protección Especial.

Ophrys corbariensis J. Samuel \& J.-M. Lewin in Orchidophile (Asnières) 154: 254, 251 (2002). Material estudiado: 
ESPAÑA. Navarra: Atez, concejo de Eguaras, Iturraitz. 30TXN0651. $640 \mathrm{~m}$. Prados de siega con Arrhenatherum elatius, margas. 13-VI-2020. $E$. Robles \& M. Becerra (PAMP 57013) (Figura 2).

Observaciones: Se trata de un taxón tardío del grupo de Ophrys scolopax Cav. descrito del sureste de Francia y que se reconoce por sus flores de gran tamaño. Entre sus principales caracteres morfológicos distintivos podemos citar los siguientes (Samuel \& Lewin, 2002): labelo cuadrangular u ovoide, de 1316 (20) mm de longitud, y aplanado en su tercio basal; gibosidades del labelo cortas; pétalos cortos y triangulares; campo basal amplio y más pálido que el labelo (color naranja ladrillo).
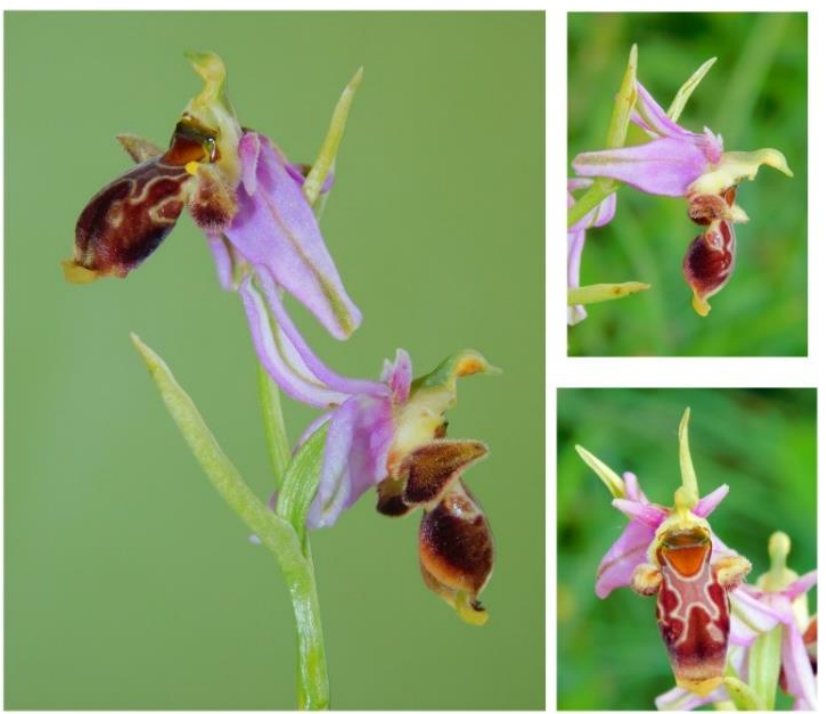

Figura 2. Ophrys corbariensis (Atez, 13-VI-2020). Figure 2. Ophrys corbariensis (Atez, 2020/06/13).

Los ejemplares observados en Navarra se corresponden con plantas robustas, de hasta $40 \mathrm{~cm}$ de alto. Inflorescencia laxa, con 5-8 flores grandes; brácteas florales de mayor longitud que los ovarios. Sépalos lanceolados, rosados, con un nervio central verdoso, dirigidos hacia atrás, superando en longitud al labelo. Pétalos laterales del mismo color que los sépalos, cortos, triangulares. Labelo grande, de $13-18 \mathrm{~mm}$ y de color marrón; mácula que supera la mitad del labelo, en forma de " $X$ ", frecuentemente compleja, prolongándose en sus extremos inferiores. Gibosidades cortas. Campo basal amplio, de color naranja ladrillo, más o menos claro, que contrasta con la tonalidad más oscura del resto del labelo.

Presenta una floración tardía, unos 15-20 días más tarde que $O$. scolopax.

Sobre su presencia en la Península Ibérica tan solo nos constan las referencias de Benito Ayuso (2017) y Hermosilla et al. (2019). El primero de ellos, al tratar la complejidad taxonómica del grupo de $O$. scolopax, indica que ha observado plantas similares a las francesas en diferentes provincias españolas. Incluye dos fotografías, una de las cuales, la perteneciente a la localidad soriana de Herreros, parece corresponderse con esta especie (labelo grande y alargado, gibosidades cortas, campo basal anaranjado y amplio). Por su parte, Hermosilla expone que la ha observado en la provincia de Burgos y en la comunidad de La Rioja.

En Navarra parece que su presencia se encuentra limitada al tercio norte de la comunidad, en zonas de clima submediterráneo de las cuencas prepirenaicas, si bien es necesario un mayor trabajo de campo para intentar conocer mejor su distribución.

Ophrys picta subsp. nafarroana E. Robles \& M. Becerra subsp. nov.

Holotypus: ESPAÑA. Navarra. Huarte, Altzutzate. 30TXN1442. $470 \mathrm{~m}$. Claros herbosos de tomillaraliagar submediterráneo, margas. 10-V-2019. E. Robles \& M. Becerra (PAMP 57020) (Figura 7.1).

Diagnosis: It differs from the type of the especies by its smaller size than the type subspecies and the inflorescence has less flowers. The petals are usually tightly triangular, rarely linear and shorter. Ovate to suborbicular lip with a rounded central lobe, not markedly strangled at the end as in Ophrys picta subsp. picta. The lip has a complex macula, shaped like " $X$ " or " $H$ ", prolonging to form open or closed circles on the sides and and at distal end, often with two small separate dots; sometimes it covers a much of the lip.

Diagnosis: se diferencia de la subespecie tipo por su menor porte e inflorescencia con menos flores. Los pétalos son en general estrechamente triangulares, rara vez lineares, más cortos. Labelo de ovado a suborbicular, con el lóbulo central redondeado, no marcadamente estrangulado en su extremo como en O. picta subsp. picta. Mácula compleja, en " $\mathrm{X}$ " o "H", que se prolonga formando círculos abiertos o no, tanto en sus laterales como en el extremo distal, frecuentemente con dos pequeños puntos separados; alguna vez llega a cubrir buena parte del labelo (Figura 3).

Descripción: plantas hasta de $20 \mathrm{~cm}$. Inflorescencia laxa, con 3-5 flores. Brácteas verdes; de mayor longitud que los ovarios. Sépalos ovado-lanceolados; los laterales agudos, de 8,5-12 x 4-6,7 mm; el central obtuso, más corto, de 6-10 × 4-7 mm; rosas, con la nerviación verde. Pétalos laterales estrechamente triangulares, rara vez lineares, con la base auriculariforme y por lo general no replegados sobre sí mismos; de 3,4-4 x 1,2-2 mm; rosáceos. Labelo de color pardo-rojizo, trilobulado, de ovado a suborbicular, de 7,6-8,6 x 4,5-5 mm; lóbulos laterales prominentes, densamente pilosos en su cara abaxial. Pseudoojos verdosos. Campo basal en forma de "U", anaranjado. Mácula compleja, en " $\mathrm{X}$ " $\mathrm{O}$ " $\mathrm{H}$ ", que se prolonga formando círculos abiertos o no, tanto en sus laterales como en el extremo distal, frecuentemente con dos pequeños puntos separados; alguna vez llega a cubrir buena parte del labelo, de tonos azulados o pardo-purpúreos y rodeada por una estrecha franja blanquecinoamarillenta. Gútula pequeña, con un diminuto apículo, dirigida hacia delante, amarillenta.

parece ser más frecuente este taxón. 
Etimología: El epíteto nafarroana hace alusión al nombre en euskera de Navarra, territorio en el que

Distribución y hábitat: En el ámbito navarro la hemos observado en las cuencas prepirenaicas de Pamplona y Aoiz-Lumbier y en la sierra de Illón (Prepirineo). Parece estar presente también en otras provincias del cuadrante noreste peninsular como Álava, Burgos, La Rioja y Zaragoza (cf. Benito Ayuso, 2017). En el sureste de Francia, si atendemos a Delforge (2018), hay plantas similares, que necesitarían un estudio más profundo para discernir si se trata del mismo taxón.

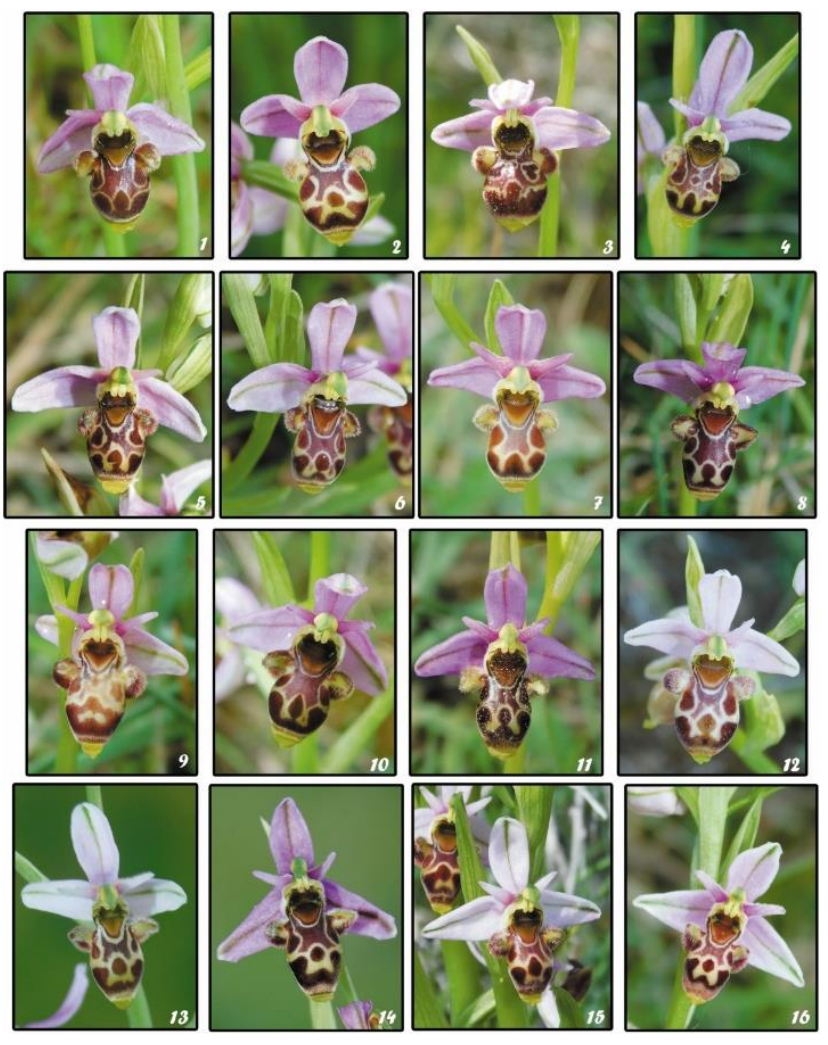

Figura 3. Ophrys picta subsp. nafarroana. 1-12) Aranguren, 19-V-2019. 13-14) Aisoáin, 29-V-2019. 15) Yesa, 15-V2019. 16) Zolina, 26-V-2019.

Figure 3. Ophrys picta subsp. nafarroana. 1-12) Aranguren, 2019/05/19. 13-14) Aisoáin, 2019/05/29. 15) Yesa, 2019/05/15. 16) Zolina, 2019/05/26.

Ocupa preferentemente tomillares y aliagares submediterráneos de Thymelaeo ruiziiAphyllanthetum monspeliensis Br.-Bi. \& $\mathrm{P}$. Montserrat. Algunas especies acompañantes son Thymus vulgaris L., Dorycnium penthaphyllum Scop., Genista scorpius (L.) DC., Genista occidentalis Rouy, Aphyllanthes monspeliensis L. y Juniperus communis L. Otras especies de orquídeas presentes son Anacamptis fragrans (Pollini) R. M. Bateman, Pridgeon \& M. W. Chase, Ophrys scolopax, O. apifera Huds., O. sphegodes Mill., O. Iutea Cav.,O. vasconica (O. Danesch \& E. Danesch) P. Delforge, Orchis anthropophora (L.) All., O. purpurea Huds., Serapias lingua L. y Gymnadenia conopsea.

Fenología: florece durante el mes de mayo.
Material estudiado complementario:

ESPAÑA. Navarra: Yesa, Valdetor. 30TXN4619. 430 m. Tomillar-aliagar, margas. 01-V-2019. E. Robles \& M. Becerra (PAMP 56177) (Figura 3.15).

ESPAÑA. Navarra. Ansoáin, laderas del Monte Ezkaba. 30TXN1143. 450 m. Herbazales, margas. 28-V-2019. E. Robles \& M. Becerra (PAMP 56185) (Figura 3.13-14).

Observaciones: la principal diferencia con la subespecie tipo está en la forma del labelo. Mientras en la subespecie picta éste es ahusado, la mayor anchura se sitúa en la parte medial y presenta un notorio estrangulamiento en su extremo distal, en la subespecie nafarroana el labelo presenta distintas morfologías, desde ovado a suborbicular u ovalado, en la mayoría de los casos con el lóbulo central de contorno, más o menos redondeando. Otros caracteres morfológicos que nos permiten separar ambos taxones son la mácula, simple en la subespecie picta y compleja en la subespecie nafarroana, y los pétalos, lineares en la primera y estrechamente triangulares en la segunda (Figura 4).

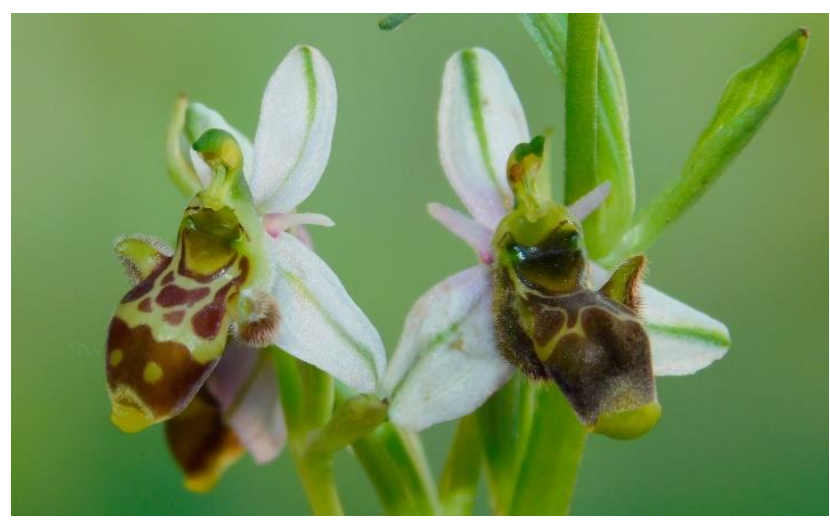

Figura 4. Comparativa entre Ophrys picta subsp. nafarroana (izquierda; Huarte, Navarra) y Ophrys picta subsp. picta (derecha; Archidona, Málaga).

Figure 4. Comparison between Ophrys picta subsp. nafarroana (left; Huarte, Navarra) and Ophrys picta subsp. picta (right; Archidona, Málaga).

La posición taxonómica de las plantas asimilables a $O$. picta presentes en la cuenca alta del Valle del Ebro y cuencas prepirenaicas ha sido controvertida. Ya a principios de los años ochenta del siglo pasado Van der Sluys \& González (1982) hacen referencia a plantas de flores pequeñas dentro del grupo de $O$. scolopax, pero no les atribuyen ninguna categoría taxonómica, aunque sí indican que deben pertenecer a una subespecie distinta.

Otros autores como Benito Ayuso (2017) muestran sus dudas sobre la presencia de $O$. picta en el Alto Ebro y el extremo septentrional del Sistema Ibérico (Álava, Burgos y La Rioja), indicando la opción de que bien pudiera tratarse de poblaciones de $O$. santonica J. M. Mathé \& Melki. Este último taxón se distingue por su floración más tardía (junio-julio), la franja amarilla que ocupa el borde del labelo, el apículo prominente que presenta la gútula y las brácteas más cortas. 
Ophrys querciphila Nicole, Hevy \& Soca in L'Orchidophile 212:94 (2017).

Material estudiado:

ESPAÑA. Navarra: Guesalaz, carretera NA-7123, ladera noroeste de Montenegro. 30TWN8728. 570 m. Herbazales en taludes de carrascal, limolitas. 30-V2019. E. Robles \& M. Becerra (PAMP 57017) (Figura 5.1).

ESPAÑA. Navarra: Guirguillano, proximidades del alto de Guirguillano.30TWN9029.760 m. Tomillares con Genista scorpius en claros de quejigal-carrascal, areniscas. 29-V-2020. (PAMP 57016) (Figura 5.2).

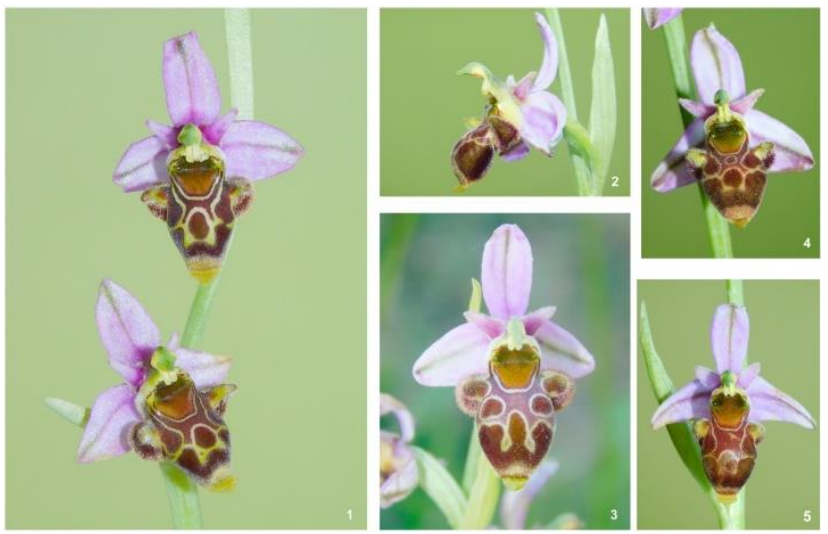

Figura 5. Ophrys querciphila. 1) Guesalaz (03-VI-2020). 25) Guirguillano (30-V-2020).

Figure 5. Ophrys querciphila. 1) Guesalaz (2020/06/03). 25) Guirguillano (2020/05/30).

Observaciones: taxón descrito del sureste de Francia que se distingue de otras especies próximas por su labelo sepioide, más rara vez scolopaxoide 0 fucifloroide, con las gibosidades poco voluminosas, y su cavidad estigmática grande y subrectangular, entre otros caracteres morfológicos (Hermosilla et al., 2019).

En cuanto a su ecología, crece en matorrales aclarados y taludes herbosos de las orlas de bosques de quercineas. En el caso de Navarra en quejigales supramediterráneos castellano-cantábricos (Spiraeo obovatae- Quercetum faginae subas. quercetosum fagineae $\mathrm{O}$. Bolòs \& $\mathrm{P}$. Montserrat) y en los carrascales castellano-cantábricos (Spiraeo obovatae-Quercetum rotundifoliae), dentro de su faciación acidófila y xerófila con Erica scoparia y Quercus coccifera. Originalmente este taxón se describió como propio de suelos calcáreos y que rehusaba los de naturaleza ácida (Nicole \& Soca, 2017). Sin embargo, lo hemos recolectado al sur del portillo de Arradia, en la umbría de Montenegro, sobre carrascales acidófilos desarrollados sobre limolitas.

Este endemismo hispano-francés del grupo de $O$. scolopax se distribuye por el sureste de Francia y de manera disyunta por cuadrante noreste peninsular (Barcelona, Burgos, Gerona, La Rioja y Navarra) (Hermosilla et al., 2019). Nuestro hallazgo supone su incorporación al catálogo florístico de Navarra (Lorda, 2013).

Su reciente descripción hace que su corología sea aún poco conocida, aunque debe de ser frecuente en la Navarra Media Oriental. Muestra de ello es una de las imágenes incluidas en el libro Orquídeas de Euskal Herria (Lizaur, 2001), en concreto la superior derecha de la página 208. Realizada en la localidad de Metauten, el autor la considera una variedad de labelo corto de 0 . scolopax pero sin duda parece corresponder al taxón que tratamos aquí.

Ophrys santonica J. M. Mathé \& F. Melki in Orchidophile (Asnières) 113:159 (1994).

[O. aestivalis J. M. Mathé \& Melki in Orchidophile (Asnières) 112:124 (1994)]; [O. scolopax subsp. santonica (J. M. Mathé \& Melki) R. Engel \& Quentin in Orchidophile (Asniéres) 124:205 (1996)]; [O. juliana M. Kerguélen in Bull. Ass. Inform. Bot. 1:24 (1994)]. Material estudiado:

ESPAÑA. Navarra: Atez, concejo de Eguaras, Iturraitz. 30TXN0651. $640 \mathrm{~m}$. Prados de siega con Arrhenatherum elatius, margas. 13-VI-2020. E. Robles \& M. Becerra (PAMP 57012) (Figura 6.2).

ESPAÑA. Navarra: Guirguillano, Muzkibidea. 30TWN9031. $706 \mathrm{~m}$. Herbazales en claros de tomillar-aliagar con Aphyllanthes monspeliensis, areniscas. 03-VI-2020. E. Robles \& M. Becerra (PAMP 57018) (Figura 6.3).

ESPAÑA. Navarra: Juslapeña, concejo de Marcaláin, Bordalar.30TXN0650.620 m. Tomillar-aliagar submediterráneo, margas. 05-VI-2020. E. Robles \& M. Becerra (PAMP57009).

ESPAÑA. Navarra: Cendea de Iza, concejo de Ariz, sierra de Zabalgaña. 30TXN0446. $480 \mathrm{~m}$. Herbazales, calcarenitas. 17-VI-2019. E. Robles \& M. Becerra (PAMP 56159).
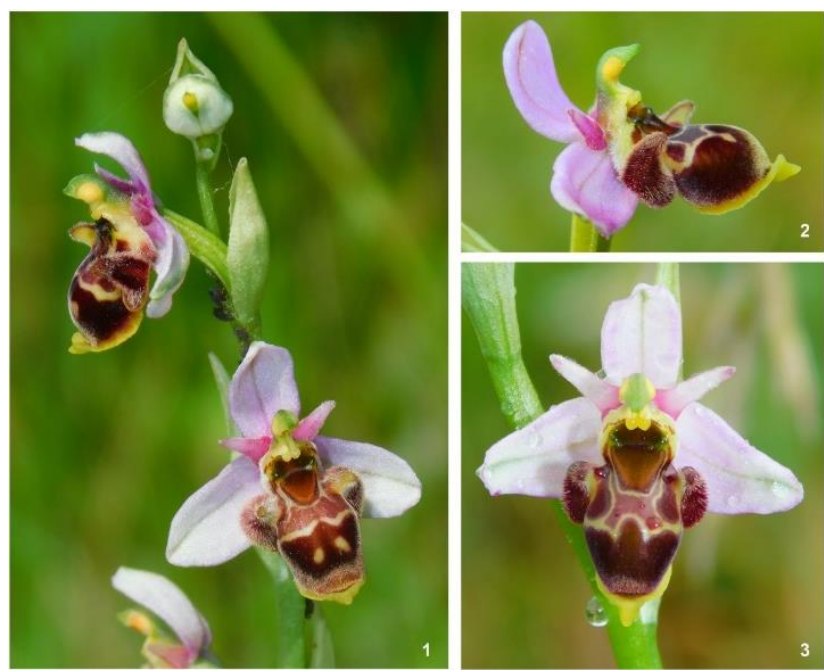

Figura 6. Ophrys santonica. 1. Castillonuevo (02-VII-2018); 2. Atez (13-VI-2020); 3. Guirguillano (03-VI-2020).

Figure 6. Ophrys santonica. 1. Castillonuevo (2018/07/02), 2. Atez (2020/06/13); 3. Guirguillano (2020/06/03).

Observaciones: taxón de floración tardía caracterizado por sus flores pequeñas y rechonchas, labelo en forma de barril con el borde amarillo y pétalos triangulares (Benito Ayuso, 2017).

En Navarra florece desde finales de mayo hasta principios de julio, observándose un gradiente 
fenológico influenciado por la altitud. Así, en cotas bajas de la Navarra Media y Cuenca de Pamplona pueden verse plantas ya en flor a finales de mayo o durante los primeros días de junio, mientras que en el Prepirineo su óptimo de floración se retrasa hasta la primera semana de julio.

Descrito con material recolectado en el oeste de Francia (Mathé \& Melki, 1994), la primera referencia para este endemismo hispano-francés en la Península Ibérica es del entorno de las localidades navarras de Bigüezal y Castillonuevo, en el Prepirineo (Thamtham et al., 2010). Con posterioridad se ha localizado en las provincias de Álava, Barcelona, Burgos, Castellón, Gerona, La Rioja y Soria (Benito Ayuso, 2017). Más recientemente se ha publicado su presencia en Guadalajara (Ramos-López et al., 2020).

En Navarra aparece de forma dispersa por el Prepirineo (sierra de Illón, proximidades del alto de las Coronas) y cuencas prepirenaicas (Cuenca de Pamplona), rarificándose hacia el sur, donde solo la hemos localizado en zonas próximas al alto de Guirguillano, en la Navarra Media. Ocupa preferentemente tomillares-aliagares submediterráneos de Thymeleo ruizii-Aphyllanthetum monspeliensis.

Ophrys x ibanezii E. Robles, M. Becerra \& A. Becerra nothosp. nov.

[O. querciphila $\times$ O. sphegodes]

Holotypus: ESPAÑA. Navarra: Guirguillano, proximidades del alto de Guirguillano. 30TWN9029. $760 \mathrm{~m}$. Tomillares con Genista scorpius en claros de quejigal-carrascal, areniscas. 29-V-2020. M. Becerra \& A. Becerra (PAMP 57021) (Figura 7.2).

Diagnosis: The lip is morphologically similar to that of Ophrys querciphila, it is a little bit wider, but it has lateral lobes and non-apiculate apical appendix smaller than $O$. querciphila. The sepals have an intermediate color tone (pinkish-green). The petals are similar to those of $O$. sphegodes but with a hairy margin, with pinkish hues.

Diagnosis: Labelo de morfología similar a $O$. querciphila, algo más ancho, pero a diferencia de ésta tiene los lóbulos laterales de menor tamaño y la gútula más pequeña y no apiculada. Los sépalos presentan una tonalidad intermedia (rosáceoverdosa). Pétalos parecidos a los de 0 . sphegodes pero con el borde piloso, con matices rosáceos.

Descripción: Plantas hasta de $35 \mathrm{~cm}$ de altura. Tallo verde, glabro. Hojas en roseta basal, lanceoladas, de 3 x $10 \mathrm{~cm}$; algunas caulinares y envainantes. Inflorescencia laxa, con hasta cinco flores. Brácteas verdes, glabras, estrechamente lanceoladas y mucho más cortas que los entrenudos, superando la longitud del ovario notablemente; de 20-35 x 4-6 mm. Sépalos de color rosado-verdoso, con el nervio central verdoso poco marcado, dirigidos hacia atrás y de $11 \times 5 \mathrm{~mm}$ de media; el central obtuso. Pétalos de subcuadrangulares a subtriangulares, con la base algo auriculariforme, verdes con matices rosáceos y con el borde piloso, hasta de 3 x 1,5 mm. Labelo de color pardo-rojizo, anchamente sepioide, trilobulado (lóbu- los laterales poco prominentes), de 10-10,4 x 6,6-6,9 $\mathrm{mm}$; prominencias poco voluminosas, densamente pilosas en su cara abaxial. Pseudoojos verdosos. Campo basal amplio, subtrapezoidal, anaranjado. Mácula en forma de "X" compleja, que se prolonga en sus extremos inferiores formando un círculo no cerrado, de tonos azulados o pardo-purpúreos y rodeada por una estrecha franja blanquecino-amari-llenta. Gútula pequeña, dirigida hacia delante, amarillenta.

Etimología: el epíteto específico está dedicado al botánico Ricardo Ibáñez Gastón, profesor de la Universidad de Navarra.

Distribución y hábitat: la única población localizada, situada en el entorno del alto de Guirguillano, estaba formada por dos individuos. Crecían en la orla de un quejigal degradado de la asociación mesosupramediterráneo subhúmedo castellano-cantábrico Spiraeo obovatae-Quercetum faginae, en claros de matorral en el que predominaba Genista scorpius Otra especie de orquídea presente en la zona es Anacamptis pyramidalis (L.) Rich.

Fenología: florece entre finales de mayo y comienzos de junio.
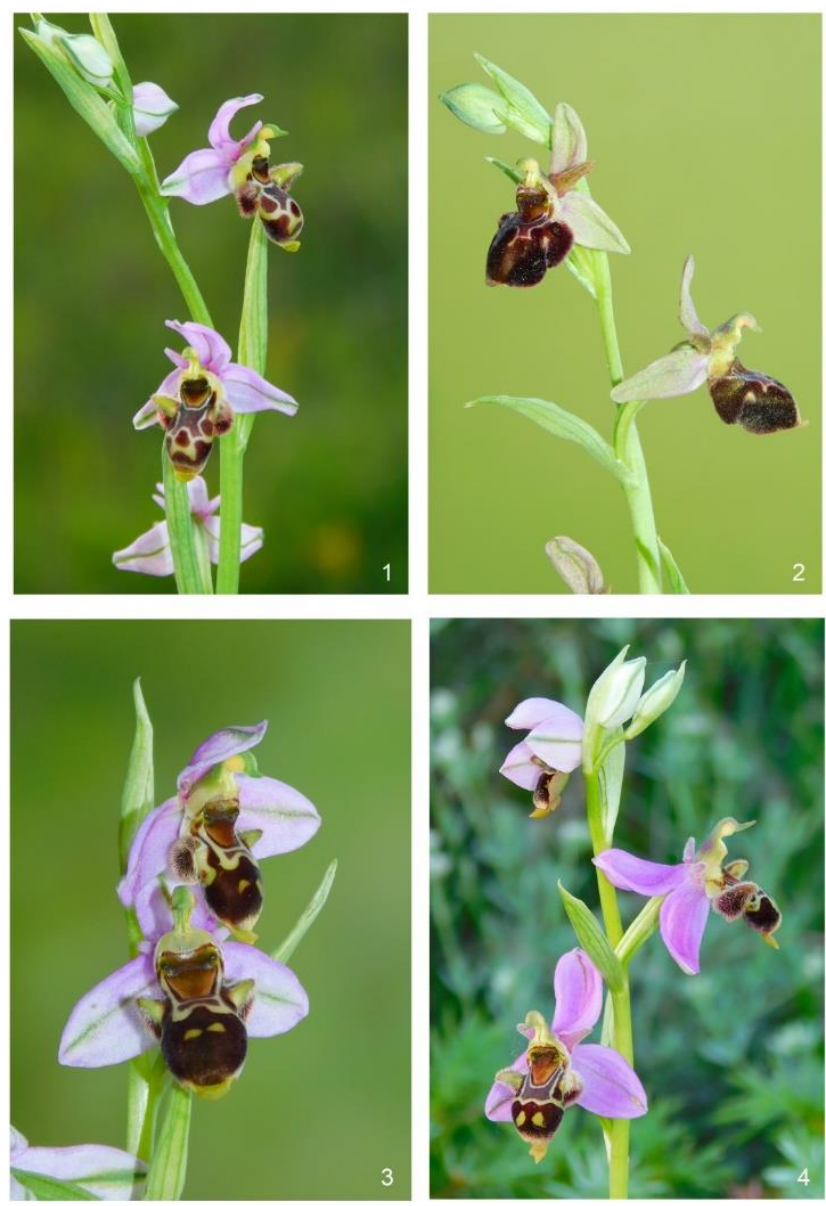

Figura 7. 1) Ophrys picta subsp. nafarroana (Huarte, 10-V2020). 2) Ophrys × ibanezii (Guirguillano, 29-V-2020). 3) Ophrys $\times$ pompelonensis (Huarte, 16-V-2020). 4) Ophrys $\times$ minuticauda (Egüés, 22-V-2020).

Figure 7. 1) Ophrys picta subsp. nafarroana (Huarte, 2020/05/10). 2) Ophrys $\times$ ibanezii (Guirguillano, 2020/05/29). 3) Ophrys $\times$ pompelonensis (Huarte, 2020/05/16). 4) Ophrys $\times$ minuticauda (Egüés, 2020/05/22). 
Ophrys $\times$ minuticauda Duffort in Bull. Vulg. Sci. Nat. 2:18,19 (1902).

[O. scolopax $\times$ O. apifera]

Material estudiado:

ESPAÑA. Navarra: Egüés, concejo de Badostáin, cerro de la Borda. 30TXN1738. $550 \mathrm{~m}$. Herbazales en claros de tomillar submediterráneo con pies sueltos de Juniperus communis y Buxus sempervirens, margas. 22-V-2020. E. Robles \& M. Becerra (PAMP 57011) (Figura 7.4).

Observaciones: Híbrido cuyo labelo presenta una morfología semejante al de $O$. apifera. Presentan algunos caracteres como las gibosidades laterales voluminosas y la gútula dirigida hacia adelante, que son propios de O. scolopax.

Tan solo nos consta una cita anterior en territorio navarro, en la localidad de Artazu, (Hermosilla \& Sabando, 1997). Con nuestra colecta ampliamos la distribución de este nothotaxon en la Comunidad Foral de Navarra.

Ophrys $\times$ pompelonensis E. Robles, M. Becerra \& A. Becerra nothosp. nov.

[O. picta subsp. nafarroana $\times O$. apifera]

Holotypus: ESPAÑA. Navarra: Huarte, cerro de Altzutzate. 30TXN1442. $470 \mathrm{~m}$. Claros herbosos de tomillar-aliagar submediterráneo, margas. 16-V2020. M. Becerra \& A. Becerra (PAMP 57022) (Figura 7.3).

Diagnosis: The lip has a size and morphology intermediate between $O$. apifera and $O$. picta subsp. nafarroana. The central sepal is not directed backwards and curved over the gynostemium. The lateral petals are similar to $O$. apifera, small and triangular but with pinkish hue as in the other parent. The apical appendix is directed forward, up to $1.6 \mathrm{~mm}$. Large greenish pseudo-eyes. The gynostemium apiculus is short, straight to slightly curved.

Diagnosis: Labelo de morfología y tamaño intermedio entre $O$. apifera y $O$. picta subsp. nafarroana. Sépalo central no dirigido hacia atrás, curvado sobre el ginostemo. Los pétalos laterales son similares a los de $O$. apifera, pequeños y triangulares, pero rosáceos como en el otro parental. Gútula dirigida hacia adelante, hasta de 1,6 mm. Pseudoojos verdosos, grandes. Apículo del ginostemo corto, de recto a ligeramente curvado.

Descripción: Plantas hasta de $18 \mathrm{~cm}$ de altura. Tallo verde, glabro. Hojas en roseta basal, lanceoladas, 6 $x 1,2 \mathrm{~cm}$; algunas caulinares y envainantes. Inflorescencia laxa, con hasta cuatro flores. Brácteas verdes, glabras, de 16-24 x 5-8 mm. Sépalos laterales rosados, con el nervio central verdoso, de $10 \times 6,6$ $\mathrm{mm}$ de media. Pétalos triangulares, rosáceos y con los márgenes recurvados, hasta de $2,5 \mathrm{~mm}$ de longitud. Labelo de color pardo-rojizo, subfusiforme, trilobulado, de $10 \times 6,3 \mathrm{~mm}$ de media. Pseudoojos verdosos. Campo basal amplio, anaranjado. Mácula en forma de "W", de tonos azulados, estrecha y rodeada por una franja amplia amarillenta, con dos pequeñas manchas amarillas separadas y en su base.
Etimología: el epíteto específico hace referencia a la ciudad romana de Pompelo, la actual Pamplona, en cuyos alrededores ha sido encontrado este híbrido.

Distribución y hábitat: tan solo hemos localizado una población integrada por cuatro individuos, en la zona oriental de la Cuenca de Pamplona. Crecían en claros herbosos de un tomillar $y$ aliagar submediterráneo de la asociación Thymelaeo ruiziiAphyllanthetum monspeliensis. Algunas especies acompañantes son Thymus vulgaris L., Dorycnium penthaphyllum Scop., Genista scorpius (L.) DC., Aphyllanthes monspeliensis L. y Juniperis communis L. Otras especies de orquídeas presentes son Anacamptis fragrans (Pollini) R. M. Bateman, Pridgeon \& M. W. Chase, O. scolopax, O. sphegodes, O. lutea Cav., Serapias lingua L. y Gymnadenia conopsea.

Fenología: florece durante la segunda quincena de mayo.

Ophrys $\times$ vistabellae J. E. Arnold in Act. Bot. Barc. 52:69 (2009).

[O. Iupercalis Devillers-Tersch.\& Devillers $\times 0$. sphegodes (sub $O$. arnoldii $\mathrm{P}$. Delforge $\times 0$. sphegodes)]

Material estudiado:

ESPAÑA. Navarra: Pamplona, Euntzetxiki. 30TXN0642. 440 m. Herbazales, margas. 06-V-2019. E. Robles \& M. Becerra (PAMP 56182) (Figura 9.2). Observaciones: Híbrido que tan solo se conocía de su localidad tipo, en la provincia de Castellón (Arnold, 2009). En la descripción original se considera como parental a $O$. arnoldii, nombre con el que se han designado en el ámbito peninsular a las plantas de floración tardía y labelo recurvado del grupo de $O$. fusca Link (Benito Ayuso, 2017). Sin embargo, muchos autores lo consideran un sinónimo de $O$. lupercalis (Benito Ayuso, 2017).

El labelo trilobulado muestra cierta similitud con el de $O$. lupercalis, así como su cavidad estigmática vallecuada, mientras que la mácula, en forma de "H" desdibujada, y un incipiente campo basal de tonalidad anaranjada son caracteres propios de $O$. sphegodes. El resto de piezas del perianto son más parecidas a las presentes en $O$. lupercalis.

Además, hemos observado este híbrido en la localidad de Eslava (alrededores de la ciudad romana de Santa Criz). Novedad para el catálogo florístico de Navarra (Lorda, 2013).

Platanthera algeriensis Bat. \& Trab. in Bull. Soc. Bot. France 39:75 (1892).

Material estudiado:

ESPAÑA. Navarra: Valle de Aranguren, concejo de Aranguren, cerro de la Borda. 30TXN1738. $540 \mathrm{~m}$. Herbazales en claros de tomillar-aliagar submediterráneo con pies sueltos de Juniperus communis y Buxus sempervirens, margas. 14-V2020. (Figura 9.1).

Observaciones: taxón distribuido por el Mediterráneo occidental (Benito Ayuso, 2017) citado por primera vez para Navarra en Castillonuevo (Thamtham et al., 
2010) y posteriormente en el Valle de Unciti (Amardeilh, 2012), es una de las orquídeas más raras de la comunidad foral. Con nuestra observación ampliamos su área de distribución a la Cuenca de Pamplona.

La población localizada en el Valle de Aranguren ocupa un hábitat diferente al de las ya conocidas de Castillonuevo y Unciti, donde aparece sobre pradosjuncales desarrollados sobre suelos húmedos o encharcados. Por el contrario, las plantas de Aranguren crecen en claros herbosos de un tomillaraliagar submediterráneo con abundantes bojes y enebros, en suelos arcillosos que se encharcan durante el invierno. Está formada por solo tres ejemplares.

Por su escasez, en 2019 contabilizamos 268 plantas en Castillonuevo y 3 en Cemboriáin (Valle de Unciti), ha sido incluida con la categoría vulnerable en el Catálogo de Especies de Flora Amenazada de Navarra (Decreto Foral 254/2019, de 16 de octubre). No se ha recolectado material por estar protegida por ley (se incluye fotografía).

Serapias $\times$ todaroi Tineo, PI. rar. Sicil. 1:12 (1846). [S. lingua L. $\times$ S. parviflora Parl.] Material estudiado:

ESPAÑA. Navarra: Ansoáin, laderas del Monte Ezkaba. 30TXN1143. $450 \mathrm{~m}$. Herbazales sobre suelos húmedos o algo encharcados en invierno, margas. 20-V-2019. E. Robles \& M. Becerra (PAMP 56162) (Figura 8).

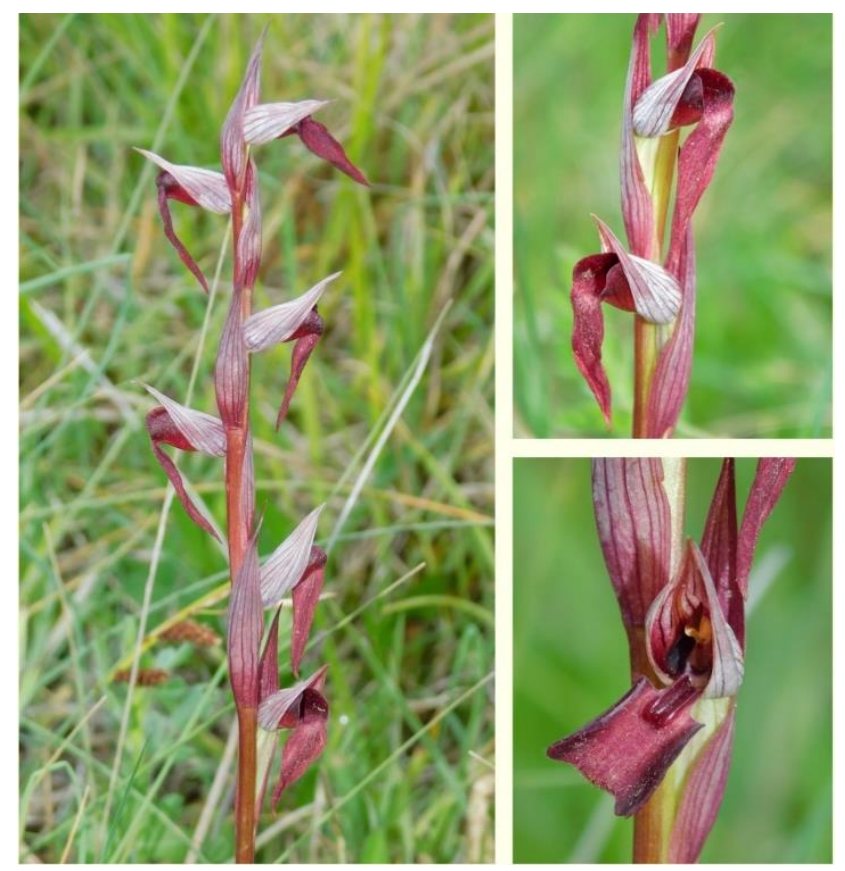

Figura 8. Serapias $\times$ todaroi (Ansoáin, 20-V-2019). 1) Inflorescencia. 2) Detalle de las flores. 3) Callosidad.

Figure 8. Serapias $\times$ todaroi (Ansoáin, 2019/05/20). 1) inflorescence. 2) Flower detail. 3) Callus detail.

Observaciones: las plantas observadas son más robustas que sus parentales y con flores muy coloreadas. La callosidad es carnosa como en $S$. lingua, estando profundamente asurcada, de tal forma que parece estar dividida en dos lamelas.

Novedad para el catálogo florístico de Navarra (Lorda, 2013), si bien debe ser frecuente a tenor de las numerosas localidades, sobre todo de la Cuenca de Pamplona, donde conviven $S$. lingua y $S$. parviflora. También la hemos observado en el término municipal de Huarte, en las campas de Ollokilanda.

Spiranthes aestivalis (Poir.) Rich., De Orchid. Eur. 36 (1817).

Material estudiado:

ESPAÑA. Navarra: Castillonuevo, Los Apaules. 30T5526. $840 \mathrm{~m}$. Prado-juncal sobre suelos encharcados, calizas. 07.VII.2019. (Figura 9.3).

Observaciones: taxón cuya área de distribución se extiende por Centroeuropa y el área mediterránea (Benito Ayuso, 2017). Es muy escaso en Navarra, donde hasta la fecha tan solo se conocían dos poblaciones en el Valle de Baztán (Balda, 2002). Ampliamos su distribución al Prepirinero, donde hemos localizado un pequeño núcleo integrado por solo cuatro plantas en la solana de la sierra de Illón.
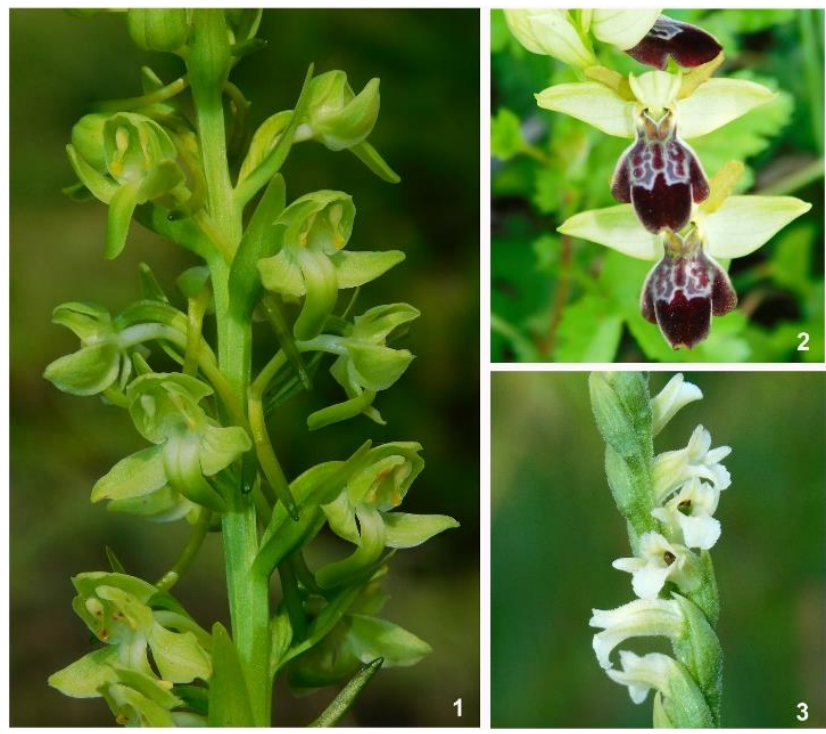

Figura 9. 1) Platanthera algeriensis (Aranguren, 14-VI2020). 2) Ophrys $\times$ vistabellae (Pamplona, 04-V-2019). 3) Spiranthes aestivalis (Castillonuevo, 07-VII-2019).

Figure 9. 1) Platanthera algeriensis (Aranguren, 2020/06/14). 2) Ophrys $\times$ vistabellae (Pamplona, 2019/05/04). 3) Spiranthes aestivalis (Castillonuevo, 2019/07/07).

En cuanto a su ecología, crecen en pradosjuncales de Epipactido palustris-Molinietum caeruleae J.-M. Montserrat, I. Soriano \& Vigo, desarrollados sobre suelos arcillosos ricos en bases que sufren un encharcamiento prolongado. Cabe destacar que este hábitat difiere del de las plantas baztanesas, pues éstas aparecen en turberas.

Por su escasez ha sido incluida con la categoría en peligro de extinción en el Catálogo de Especies de Flora Amenazada de Navarra (Decreto Foral 254/2019, de 16 de octubre). 
No se ha recolectado material por estar protegida por ley (se incluye fotografía).

\section{Agradecimientos}

Al Servicio de Biodiversidad del Departamento de Desarrollo Rural y Medio Ambiente del Gobierno de Navarra por las facilidades para la obtención de la autorización para los muestreos de orquideoflora en la Comunidad Foral de Navarra. A Ricardo lbáñez por la lectura crítica del manuscrito y sus aportaciones que han enriquecido el texto. A Alberto Martínez Saldaña por sus apreciaciones a las diagnosis en inglés. A José Ardaiz por sus indicaciones sobre la localización de Gymnadenia pyrenaica en Navarra.

\section{Bibliografía}

Aedo, C. (2005). Gymnadenia R. Br. In C. Aedo \& A. Herrero (eds.) (2005). Flora iberica vol. XXI Smilaceae-Orchidaceae (pp 82-85). Madrid: Real Jardín Botánico, CSIC.

Aizpuru, I., C. Aseginolaza, P. M. Uribe-Echebarría, P. Urrutia \& I. Zorrakin (2000). Claves ilustradas de la flora del País Vasco y territorios limítrofes. Servicio de Publicaciones del Gobierno Vasco.

Amardeilh, J. P. (2012). Orchids of Navarre (Spain) Distribution and mapping. J. Eur. Orch., 44(2), 235-336.

Arnold. J. E. (2009). Notes sobre el gènere Ophrys L. (Orchidaceae) a Catalunya i al País Valencià. Acta Bot. Barc., 52, 45-82.

Balda, A. (2002). Contribuciones al conocimiento de la flora navarra. Munibe, 53, 157-174.

Benito Ayuso, J. (2017). Estudio de las orquídeas silvestres del Sistema Ibérico. Universitat de València. https://roderic.uv.es/handle/10550/59567.

Delforge, P. (2018). Guía de campo de las orquídeas de Europa, África del Norte y Oriente Próximo. Omega.

Hermosilla, C. E. (2000). Notas sobre orquídeas (VII). Est. Mus. Cienc. Nat. de Álava, 15, 189-208.

Hermosilla Fernández, C. E., J. Pérez Cañestro \& R. Soca (2019). Ophrys querciphila Nicole, Hervy \& Soca en la Península Ibérica. Flora Montiberica, 75, 67-72.
Hermosilla, C. E. \& J. Sabando (1996). Notas sobre orquídeas (II). Est. Mus. Cienc. Nat. Álava, 10-11, 119-140.

Hermosilla, C. E. \& J. Sabando (1997). Notas sobre orquídeas (IV). Est. Mus. Cienc. Nat. Alava, 12, 57-68.

Lizaur, X. (2001). Orquídeas de Euskal Herria. Servicio Central de Publicaciones del Gobierno Vasco.

Lorda López, M. (2013). Catálogo Florístico de Navarra. Monografías de Botánica Ibérica 11. Pamplona: Jolube.

Mathé, J. M. \& F. Melki (1994). Ophrys aestivalis une nouvelle espéce à floration tardive dans le centreouest de la France. L'Orchidophile (Asniéres), 112, 120-126.

Mathé, J. M. \& F. Melki (1994).Ophrys santonica un nouveau nom valide por Ophrys aestivalis Mathé \& Melki. L'Orchidophile (Asniéres), 113, 158-159.

Nicole, M. \& R. Soca (2017). Ophrys querciphila Nicole, Hervy \& Soca sp. nova, Ophrys tardif du Languedoc. L'Orchidophile (Asniéres), 212, 89-99.

Peralta, J., I. Biurrun, I. García-Mijangos, J. L. Remón, J. M. Olano, M. Lorda, J. Loidi \& J. Campos (2018). Manual de hábitats de Navarra. Departamento de Desarrollo Rural, Medio Ambiente y Administración Local. Gobierno de Navarra.

Ramos-López, B., J. Tobajas González \& J. Roca Juncosa (2020). Primera cita de Ophrys santonica J. M. Mathé y F. Melki (Orchidaceae) en la provincia de Guadalajara. Reduca (Biología). Serie Botánica, 13(1), 1-9.

Samuel, J. \& J.-M.Lewin (2002). Ophrys corbariensis J. Samuel \& J.-M.Lewin, sp. nova, Ophrys tardif du groupe scolopax. L'Orchidophile (Asniéres), 154, 251-258.

Sánchez Pedraja, Ó. (2005). Dactylorhiza Neck. ex Nevski. In C. Aedo. \& A. Herrero (Eds.) Flora iberica vol. XXI Smilaceae-Orchidaceae (pp. 94111). Madrid: Real Jardín Botánico, CSIC.

Thamtham, M., J. Puyo \& M. Saule (2010). Ophrys santonica J. M. Mathé \& Melki, orchidée nouvelle pour la Navarre espagnole. Le Monde des Plantes, 501, 27-29.

Van der Sluys, M. \& J. González Atarbe (1982). Orquídeas de Navarra. Diputación Provincial de Navarra. Institución Príncipe de Viana. 